\title{
OBSERVED LITHIUM ABUNDANCES AS A TEST OF STELLAR INTERNAL STRUCTURE
}

\author{
Sylvie Vauclair - Observatoire Midi-Pyrénées - France
}

The "lithium gap" observed in the Hyades and other galactic clusters by Ann Boesgaard and her collaborators (Boesgard and Tripicco 1986, Boesgaard 1987, Boesgaard, Budge and Burck 19871 gives a challenge to theoreticians. Indeed a good fit between the theoretical results and the observations will give, a clue for our understanding of the stellar internal structure and evolution.

A theoretical explanation of the "lithium gap" by gravitational and radiative diffusion has been proposed by Michaud 1986. In $G$ type stars, the convection zone is too deep for gravitational settling to take place : the density at the bottom of the convection zone is so large that the diffusion time scale exceeds the age of the star. Increasing the effective temperature leads to a decrease of the convection zone, and consequently to a decrease of the diffusion time scale. In $F$ stars it becomes smaller than the stellar age, leading qualitatively to a lithium abundance decrease as observed. When the convection zone is shallow enough, the radiative acceleration on lithium becomes important as lithium is in the hydrogenic form of li III (while it is a bare nucleus, li IV, deeper in the star). This radiative acceleration may prevent lithium settling for hotter $F$ stars. This is a very attractive explanation, which leads to a minimum of the lithium abundance nearly at the place where it is observed in effective temperature. However it suffers from some difficulties : the theory predicts an increase of the lithium abundance larger than normal in the hottest $F$ stars, which is not observed, and the predicted minimum lithium abundance is one or two orders of magnitude higher than the minimum observed in the Hyades. The former may be overcome if mass loss occurs in these stars (Michaud 861 . Let us focus on the latter.

The radiative acceleration on a given element, through a bound-bound transition, may be written:

$g_{R}=\frac{1}{m} \frac{N_{i, n}}{N} \int_{0}^{\infty} \sigma_{i, n}(v) \frac{\phi v d v}{c}$

where $m$ is the mass of the considered element, $N_{i}, N$ the fraction of the element in the lower level of the line, $\sigma_{i, n}(v)$ the transition section and $\phi_{v} d v$ the available photon flux.

With the diffusion approximation, a lorentz profile for the line, and after integration over $v, g_{R}$ becomes :

$g_{R}=\frac{1}{m N} \frac{8 \pi^{2} k^{3}}{3 h^{2} c^{3}} T^{2}\left(-\frac{d T}{d r}\right) \frac{z^{4}-e^{z}}{\left(e^{z}-1\right)^{2}} \frac{\Delta / 2}{\sqrt{\frac{k_{C}}{k_{L}}\left(\frac{k_{C}}{k_{L}}+1\right)}}$

with $z=\frac{h v}{k T} \quad$ and $k_{L}=N_{n} \frac{\pi e^{2}}{m_{e} c} \quad \frac{f}{\pi} \frac{2}{\Delta}$

where $f$ is the oscillator strength of the line and $\Delta / 2$ the half width. ${ }^{k} c$ is the monochromatic opacity due to all the opacity sources except the considered line.

For an unsaturated line $\left(k_{L}<\left\langle k_{C}\right),(2)\right.$ may be transfarmed into:

$g_{R}=\frac{1.6 \times 10^{-4}}{A} \frac{N_{i, n}}{N}+\frac{z^{4} e^{z}}{\left(e^{z}-1\right)^{2}} \frac{T_{e}^{4}}{T} \frac{R^{2}}{r^{2}} \frac{\bar{k}}{k_{c}}$

where $\bar{k}$ is the Rosseland mean opacity. Te the effective temperature, $T$ the local temperature, $R$ the stellar radius, $r$ the local radius

The ratio $\bar{k} / k_{c}$ which appears in $g_{R}$ represents the fact that the radiative acceleration through one line strongly depends on the other sources of opacity at the same frequency. Up to now, the radiative accelerations have been computed with the approximation $k_{c} \approx \bar{k}$. However if, for example, a line of an abundant element sits at the same place as the Ly a lithium resonance line, the radiative acceleration on lithium may be strongly decreased.

A table of the important lines which may blend the lithium and beryllium resonance lines has been given in vauclair 1987. This table is not exhaustive as this 
part of the spectrum is not well known. Also the atomic parameters of these lines are very uncertain. The influence of these lines on the lithium and beryllium radiative accelerations is shown on fig. 1. It seems too small to account for the discrepancy between the theoretical and observed minima of $L i$ abundances. However these results are very uncertain as this part of the spectrum is not well known. I would like to emphasize the urgent need of good atomic parameters for these far uv 1 ines.

Another explanation of the lithium gap in the Hyades could be found in terms of turbulent diffusion and nuclear destruction. Turbulence is definitely needed to explain the lithium abundance decrease in $G$ stars. If this turbulence is due to the shear flow instability induced by meridional circulation lBaglin. Morel, Schatzman 1985, Zahn 1983), turbulence should also occur in F stars, which rotate more rapidly than G stars. Fig. 2 shows a comparison between the turbulent diffusion coefficient needed for lithium nuclear destruction and the one induced by turbulence. Li should indeed be destroyed in $F$ stars: This effect gives an alternative scenario to account for the Li gap in the Hyades. The fact that $L i$ is normal in the hottest observed $F$ stars could be due to their slow rotation.

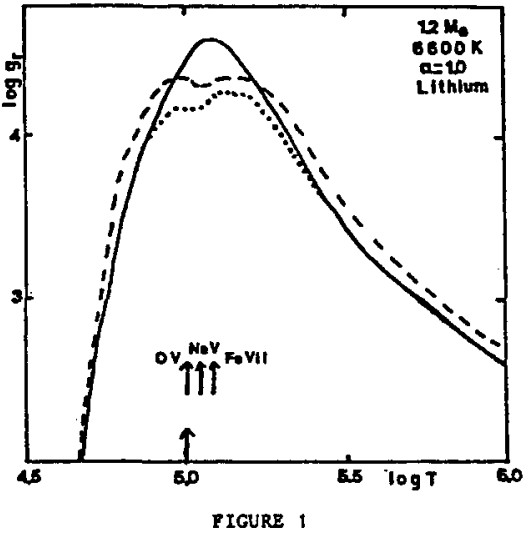

Radiative acceleration on $L i$ in $\$ 1.2 . H_{Q}$ sear.

$\begin{array}{ll}- & k_{v}=\bar{x} \\ \ldots & k_{v}=\bar{k}+\text { opacity of blendings lines } \\ -- & k_{v}=\bar{x} / 2+\text { opacity of blending lines }\end{array}$

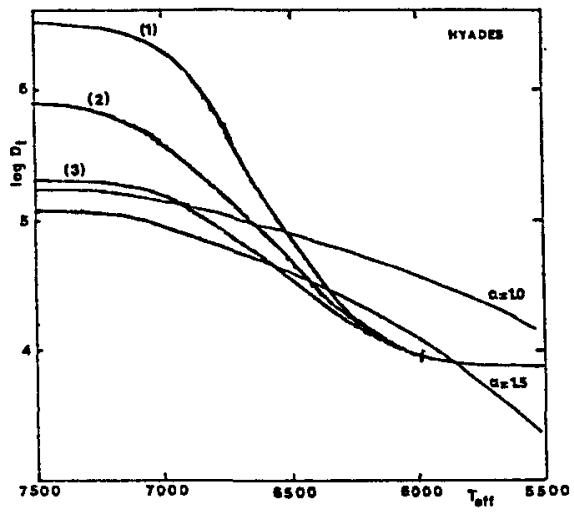

FIGURE 2

Turbulent diffusion coefficients.

-curves labelled $a=1.0$ and $a=1.5: D_{Y}$ needed for Li nuclear destruction (a) mixing length parameter)

-curves (1), (2), (3): $D_{T}$ induced by rotation (Zahn 1983). these curves are adjusted for $\mathrm{Li}$ nuclear destruction in olar type tars.

(1): present rotacion law; (2): assuming Endal and Sofia 1981 braking; (3): assuming Skumanitch 1972 braking.

\section{References}

Baglin,A., Marel,P., Schatzman,E. 1985, Astron. Astrophys. 149, 309

Benz,W. Mayor,M. Mermilliod,J.C. 1984. Astron. Astrophys. 138, 93

Boesgaard,A.M. Tripicco,M.J. 1986, ApJ 302, L49

Boesgaard,A.M., Budge,K.G., Burck,E.E. 1987, preprint

Boesgaard,A.M. 1987, P.A.S.P., in press

Boesgaard,A.M. 1987, ApJ, in press

Endal,A.S., Sofia,S. 1981, Ap. J. 243, 625

Michaud, G. 1986, ApJ. 302, 650

Skumanitch, A. 1972, Ap. J. 171, 565

Vauclair,S. 1987, proceedings of the IAU symposium 132 "The impact of very high s/N spectroscopy on stellar physics".

Zahn, J.-P. 1983, in "Saas Fee advanced courses in Astrophysics". 